\title{
What Cannot Be Computed Locally!
}

\section{Report}

Author(s):

Kuhn, Fabian; Moscibroda, Thomas; Wattenhofer, Roger

Publication date:

2004

Permanent link:

https://doi.org/10.3929/ethz-a-006741901

Rights / license:

In Copyright - Non-Commercial Use Permitted

Originally published in:

Technical Report / ETH Zurich, Department of Computer Science 438 


\title{
What Cannot Be Computed Locally!
}

\author{
Fabian Kuhn, Thomas Moscibroda, Roger Wattenhofer \\ \{kuhn@inf, moscitho@student, wattenhofer@inf. ethz.ch
}

\begin{abstract}
We give time lower bounds for the distributed approximation of minimum vertex cover (MVC) and related problems such as minimum dominating set (MDS). In $k$ communication rounds, MVC and MDS can only be approximated by factors $\Omega\left(n^{c / k^{2}} / k\right)$ and $\Omega\left(\Delta^{1 / k} / k\right)$ for some constant $c$, where $n$ and $\Delta$ denote the number of nodes and the largest degree in the graph. The number of rounds required in order to achieve a constant or even only a polylogarithmic approximation ratio is at least $\Omega(\sqrt{\log n / \log \log n})$ and $\Omega(\log \Delta / \log \log \Delta)$. By a simple reduction, the latter lower bounds also hold for the construction of maximal matchings and maximal independent sets.
\end{abstract}

Keywords: locality, lower bounds, local computation, distributed algorithms, complexity

\section{Introduction and Related Work}

What can be computed locally? Naor and Stockmayer [15] raised this for the distributed computing community vital question over a decade ago, and though the price of locality has puzzled researchers ever since, results have been rare [12]. The importance of locality stems from the desire of achieving a global goal based on local information only. This is not only one of the key challenges when developing fast distributed algorithms, but it also improves faulttolerance and allows detecting illegal global configurations by checking local conditions [1]. In light of recent advances in networking and ever-growing distributed systems, the need for a thorough understanding of locality-issues is urging. In the present paper, we study the impact of locality in the classic message passing model where the distributed system is modelled as a graph: Nodes represent the processors and two nodes can communicate if and only if they share an edge in the graph.

We present lower bounds for traditional graph theory problems, starting out with minimum vertex cover (MVC). A vertex cover for graph $G=(V, E)$ is a subset of nodes $V^{\prime} \subseteq V$ such that, for each edge $(u, v) \in E$, at least one of the two incident nodes $u, v$ belongs to $V^{\prime}$. Finding a vertex cover with minimum cardinality is known as the MVC problem.

In local algorithms, nodes are only allowed to communicate with their direct neighbors in $G$. After several rounds of communication they need to come up with a global solution, e.g. a good approximation for MVC. Intuitively MVC could be considered perfectly suited for a local algorithm: A node should be able to decide whether to join the vertex cover by communicating with its neighbors a few times. Very distant nodes seem to be superfluous for this decision. The fact that there is a simple greedy algorithm which approximates MVC within a factor 2 in the global setting, additionally raises hope for an efficient local algorithm.

To our surprise, however, there is no such algorithm. In this paper, we show that in $k$ communication rounds, MVC can only be approximated by a factor $\Omega\left(n^{c / k^{2}} / k\right)$ for a constant $c$ larger than $1 / 4$. This implies a running time of $\Omega(\sqrt{\log n / \log \log n})$ in order to achieve a constant or even polylogarithmic approximation ratio. When making the result dependent on the maximum degree $\Delta$ instead of the number of nodes $n$, the approximation ratio is $\Omega\left(\Delta^{1 / k} / k\right)$ and the time needed to obtain a polylogarithmic or constant approximation is $\Omega(\log \Delta / \log \log \Delta)$. The results for MVC can be extended to minimum dominating set (MDS) and other distributed covering problems. Since these lower bounds hold even in the cases of unbounded 
messages and complete synchrony, the lower bounds are a true consequence of locality limitations, and not merely a side-effect of congestion, asynchrony, or limited message size. Moreover, some of our lower bounds are almost tight [8].

A pioneering and seminal lower bound by Linial [12] showed that the non-uniform $\mathrm{O}\left(\log ^{*} n\right)$ coloring algorithm by Cole and Vishkin [2] is asymptotically optimal for the ring. This lower bound has been cherished by researchers as a fundamental advancement in the theory of distributed computation. To the best of our knowledge, it is the only general lower bound for local computation [4].

Linial's lower bound is based on the drosophila melanogaster of distributed computing, the ring network. For MVC, highly symmetric graphs such as rings often feature a straight-forward solution with constant approximation ratio. In any $\delta$-regular graph, for example, the algorithm which includes all nodes in the vertex cover is already a 2-approximation for MVC: Each node will cover at most $\delta$ edges, the graph has $n \delta / 2$ edges, and therefore at least $n / 2$ nodes need to be in the minimum vertex cover. On the other extreme, asymmetric graphs often enjoy constant-time algorithms, too. In a tree, choosing all inner nodes yields a 2-approximation. The same trade-off exists for node degrees. If the maximum node degree is low (constant), we can tolerate to choose all nodes, and have by definition a good (constant) approximation. If there are nodes with high degree, the diameter of the graph is small, and a few communication rounds suffice to inform all nodes of the entire graph. What we need is a construction of a not too symmetric and not too asymmetric graph with a variety of node degrees! Not many graphs with these "non-properties" are known in distributed computing.

The proof of our lower bounds is based on the timeless indistinguishability argument [5,9]. In $k$ rounds of communication, a network node can only gather information about nodes which are at most $k$ hops away and hence, only this information can be used to determine the computation's outcome. In particular, we show that after $k$ communication rounds two neighboring nodes see exactly the same graph topology; informally speaking, both neighbors are equally qualified to join the vertex cover. How- ever, in our example graphs, choosing the wrong neighbor will be ruinous.

The remainder of the paper is organized as follows. Section 2 describes the model of computation. The lower bound is constructed in Section 3. In Section 4, we show how our MVC lower bound can be extended to minimum dominating set (MDS), maximal matching (MM), and maximal independent set (MIS). Section 5 concludes the paper.

\section{Model}

We consider the classic message passing model, which consists of a point-to-point communication network, described by an undirected graph $G=$ $(V, E)$. Nodes correspond to processors and edges represent communication channels between them. In one communication round, each node of the network graph can send an arbitrarily long message to each of its neighbors. Local computations are for free and initially, nodes have no knowledge about the network graph. They only know their own unique identifier ${ }^{1}$. In $k$ communication rounds, a node $v$ may collect the IDs and interconnections of all nodes up to distance $k$ from $v . \mathcal{T}_{v, k}$ is defined to be the topology seen by $v$ after these $k$ rounds, i.e. $\mathcal{T}_{v, k}$ is the graph induced by the $k$-neighborhood of $v$. The labelling (i.e. the assignment of IDs) of $\mathcal{T}_{v, k}$ is denoted by $\mathcal{L}\left(\mathcal{T}_{v, k}\right)$.

Because message size is unbounded, the best a deterministic algorithm can do in time $k$, is to collect its $k$-neighborhood and base its decision on $\left(\mathcal{T}_{v, k}, \mathcal{L}\left(\mathcal{T}_{v, k}\right)\right)$. In other words, a deterministic distributed algorithm can be regarded as a function mapping $\left(\mathcal{T}_{v, k}, \mathcal{L}\left(\mathcal{T}_{v, k}\right)\right)$ to the possible outputs. For randomized algorithms, we apply the standard trick that all random bits can be determined at the beginning of the algorithm. In this case, a node $v$ additionally knows about the random bits $\mathcal{R}\left(\mathcal{T}_{v, k}\right)$ of all nodes in $\mathcal{T}_{v, k}$. Hence, a randomized algorithm is a deterministic function which assigns a valid output value to each triple $\left(\mathcal{T}_{v, k}, \mathcal{L}\left(\mathcal{T}_{v, k}\right), \mathcal{R}\left(\mathcal{T}_{v, k}\right)\right)$.

The model presented is in accordance with the one used in [12] and textbooks [16]. It is the strongest possible model when proving lower bounds for local

\footnotetext{
${ }^{1}$ Our results hold for any possible ID space including the standard case where IDs are the numbers $1, \ldots, n$.
} 
computations because it focuses entirely on the locality of distributed problems and abstracts away other issues arising in the design of distributed algorithms (e.g. need for small messages, fast local computations, etc.). This guarantees that our lower bounds are true consequences of locality.

\section{Lower Bound}

We first give an outline of the proof. The basic idea is to construct a graph $G_{k}=(V, E)$, for each positive integer $k$, which contains a bipartite subgraph $S$ with node set $C_{0} \cup C_{1}$ and edges in $C_{0} \times C_{1}$ as shown in Figure 1. Set $C_{0}$ consists of $n_{0}$ nodes each of which has $\delta_{0}$ neighbors in $C_{1}$. Each of the $n_{0} \cdot \frac{\delta_{0}}{\delta_{1}}$ nodes in $C_{1}$ has $\delta_{1}, \delta_{1}>\delta_{0}$, neighbors in $C_{0}$. The goal is to construct $G_{k}$ in such a way that all nodes in $v \in S$ see the same topology $\mathcal{T}_{v, k}$ within distance $k$. In a globally optimal solution, all edges of $S$ may be covered by nodes in $C_{1}$ and hence, no node in $C_{0}$ needs to join the vertex cover. In a local algorithm, however, the decision of whether or not a node joins the vertex cover depends only on its local view, i.e. the triple $\left(\mathcal{T}_{v, k}, \mathcal{L}\left(\mathcal{T}_{v, k}\right), \mathcal{R}\left(\mathcal{T}_{v, k}\right)\right)$. We show that because adjacent nodes in $S$ see the same $\mathcal{T}_{v, k}$, every algorithm adds a large portion of nodes in $C_{0}$ to its vertex cover in order to end up with a feasible solution. In other words, we construct a graph in which the symmetry between two adjacent nodes cannot be broken within $k$ communication rounds. This yields suboptimal local decisions and hence, a suboptimal approximation ratio. Throughout the proof, we will use $C_{0}$ and $C_{1}$ to denote the two sets of the bipartite subgraph $S$.

Our proof is organized as follows. The structure of $G_{k}$ is defined in Subsection 3.1. In Subsection 3.2, we show how $G_{k}$ can be constructed without small cycles, ensuring that each node sees a tree within distance $k$. Subsection 3.3 proves that adjacent nodes in $C_{0}$ and $C_{1}$ have the same view $\mathcal{T}_{v, k}$ and finally, Subsection 3.4 derives the lower bounds.

\subsection{The Cluster Tree}

The nodes of graph $G_{k}=(V, E)$ can be grouped into disjoint sets which are linked to each other as bipartite graphs. We call these disjoint sets of nodes clusters.

We define the structure of $G_{k}$ using a directed tree $C T_{k}=(\mathcal{C}, \mathcal{A})$ with doubly labelled $\operatorname{arcs} \ell: \mathcal{A} \rightarrow$ $\mathbb{N} \times \mathbb{N}$. We refer to $C T_{k}$ as the cluster tree, because each vertex $C \in \mathcal{C}$ represents a cluster of nodes in $G_{k}$. The size of a cluster $|C|$ is the number of nodes the cluster contains. An arc $a=(C, D) \in \mathcal{A}$ with $\ell(a)=\left(\delta_{C}, \delta_{D}\right)$ denotes that the clusters $C$ and $D$ are linked as a bipartite graph, such that each node $u \in C$ has $\delta_{C}$ neighbors in $D$ and each node $v \in D$ has $\delta_{D}$ neighbors in $C$. It follows that $|C| \cdot \delta_{C}=$ $|D| \cdot \delta_{D}$. We call a cluster leaf-cluster if it is adjacent to only one other cluster, and we call it inner-cluster otherwise.

Definition 3.1. The cluster tree $C T_{k}$ is recursively defined as follows:

$$
\begin{aligned}
C T_{1} & :=\left(\mathcal{C}_{1}, \mathcal{A}_{1}\right), \quad \mathcal{C}_{1}:=\left\{C_{0}, C_{1}, C_{2}, C_{3}\right\} \\
\mathcal{A}_{1} & :=\left\{\left(C_{0}, C_{1}\right),\left(C_{0}, C_{2}\right),\left(C_{1}, C_{3}\right)\right\} \\
\ell\left(C_{0}, C_{1}\right) & :=\left(\delta_{0}, \delta_{1}\right), \quad \ell\left(C_{0}, C_{2}\right):=\left(\delta_{1}, \delta_{2}\right), \\
\ell\left(C_{1}, C_{3}\right) & :=\left(\delta_{0}, \delta_{1}\right)
\end{aligned}
$$

Given $C T_{k-1}$, we obtain $C T_{k}$ in two steps:

- For each inner-cluster $C_{i}$, add a new leafcluster $C_{i}^{\prime}$ with $\ell\left(C_{i}, C_{i}^{\prime}\right):=\left(\delta_{k}, \delta_{k+1}\right)$.

- For each leaf-cluster $C_{i}$ of $C T_{k-1}$ with $\left(C_{p}, C_{i}\right) \in \mathcal{A}$ and $\ell\left(C_{p}, C_{i}\right)=\left(\delta_{p}, \delta_{p+1}\right)$, add $k-1$ new leaf-clusters $C_{j}^{\prime}$ with $\ell\left(C_{i}, C_{j}^{\prime}\right):=$ $\left(\delta_{j}, \delta_{j+1}\right)$ for $j=0 \ldots k, j \neq p+1$.

Further, we define $\left|C_{0}\right|=n_{0}$ for all $C T_{k}$.

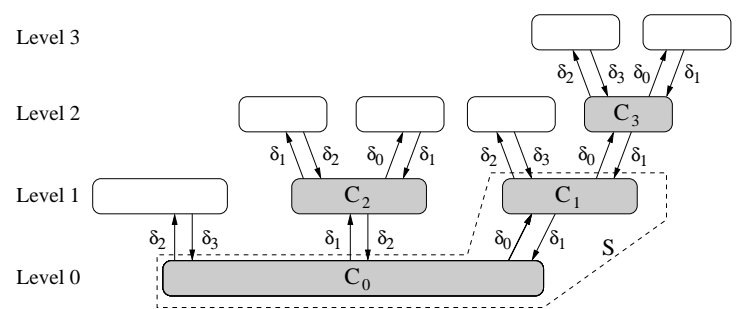

Figure 1: Cluster-Tree $C T_{2}$.

Figure 1 shows $C T_{2}$. The shaded subgraph corresponds to $C T_{1}$. The labels of each arc $a \in \mathcal{A}$ are of the form $\ell(a)=\left(\delta_{l}, \delta_{l+1}\right)$ for some $l \in\{0, \ldots, k\}$. 
Further, setting $\left|C_{0}\right|=n_{0}$ uniquely determines the size of all other clusters. In order to simplify the upcoming study of the cluster tree, we need two additional definitions. The level of a cluster is the distance to $C_{0}$ in the cluster tree (cf. Figure 1). The depth of a cluster $C$ is its distance to the furthest leaf in the subtree rooted at $C$. Hence, the depth of a cluster plus one equals the height of the subtree corresponding to $C$. In the example of Figure 1, the depths of $C_{0}, C_{1}, C_{2}$, and $C_{3}$ are $3,2,1$, and 1 , respectively.

Note that $C T_{k}$ describes the general structure of $G_{k}$, i.e. it defines for each node the number of neighbors in each cluster. However, $C T_{k}$ does not specify the actual adjacencies. In the next subsection, we show that $G_{k}$ can be constructed so that each node's view is a tree.

\subsection{The Lower Bound Graph $G_{k}$}

In Subsection 3.3, we will prove that the topologies seen by nodes in $C_{0}$ and $C_{1}$ are identical. This task is greatly simplified if each node's topology is a tree (rather than a general graph) because we do not have to worry about cycles. The girth of a graph $G$, denoted by $g(G)$, is the length of the shortest cycle in $G$. We want to construct $G_{k}$ with girth at least $2 k+1$ so that in $k$ communication rounds, all nodes see a tree. Given the structural complexity of $G_{k}$ for large $k$, constructing $G_{k}$ with large girth is not a trivial task. The solution we present is based on the construction of the graph family $D(r, q)$ as proposed in [10]. For given $r$ and $q, D(r, q)$ defines a bipartite graph with $2 q^{r}$ nodes and girth $g(D(r, q)) \geq r+5$. In particular, we show that for appropriate $r$ and $q$, we obtain an instance of $G_{k}$ by deleting some of the edges of $D(r, q)$. In the following, we introduce $D(r, q)$ up to the level of detail which is necessary to understand our results. For the interested reader, we refer to [10].

For an integer $r \geq 1$ and a prime power $q, D(r, q)$ defines a bipartite graph with node set $P \cup L$ and edges $E_{D} \subset P \times L$. The nodes of $P$ and $L$ are labelled by the $r$-vectors over the finite field $\mathbb{F}_{q}$, i.e. $P=L=\mathbb{F}_{q}^{r}$. In accordance with [10], we denote a vector $p \in P$ by $(p)$ and a vector $l \in L$ by $[l]$. The components of $(p)$ and $[l]$ are written as follows (for $D(r, q)$, the vectors are projected onto the first $r$ coordinates):

$$
\begin{aligned}
(p)= & \left(p_{1}, p_{1,1}, p_{1,2}, p_{2,1}, p_{2,2}, p_{2,2}^{\prime}, p_{2,3}, p_{3,2}, \ldots\right. \\
& \left.p_{i, i}, p_{i, i}^{\prime}, p_{i, i+1}, p_{i+1, i}, \ldots\right) \\
{[l]=} & {\left[l_{1}, l_{1,1}, l_{1,2}, l_{2,1}, l_{2,2}, l_{2,2}^{\prime}, l_{2,3}, l_{3,2}, \ldots\right.} \\
& \left.l_{i, i}, l_{i, i}^{\prime}, l_{i, i+1}, l_{i+1, i}, \ldots\right] .
\end{aligned}
$$

Note that the somewhat confusing numbering of the components of $(p)$ and $[l]$ is chosen in order to simplify the following system of equations. There is an edge between two nodes $(p)$ and $[l]$, exactly if the first $r-1$ of the following conditions hold (for $i=2,3, \ldots)$.

$$
\begin{aligned}
l_{1,1}-p_{1,1} & =l_{1} p_{1} \\
l_{1,2}-p_{1,2} & =l_{1,1} p_{1} \\
l_{2,1}-p_{2,1} & =l_{1} p_{1,1} \\
l_{i, i}-p_{i, i} & =l_{1} p_{i-1, i} \\
l_{i, i}^{\prime}-p_{i, i}^{\prime} & =l_{i, i-1} p_{1} \\
l_{i, i+1}-p_{i, i+1} & =l_{i, i} p_{1} \\
l_{i+1, i}-p_{i+1, i} & =l_{1} p_{i, i}^{\prime}
\end{aligned}
$$

In [10], it is shown that for odd $r \geq 3, D(r, q)$ has girth at least $r+5$. Further, if a node $u$ and a coordinate of a neighbor $v$ is fixed, the remaining coordinates of $v$ are uniquely determined. This is concretized in the next lemma.

Lemma 3.2. For all $(p) \in P$ and $l_{1} \in \mathbb{F}_{q}$, there is exactly one $[l] \in L$ such that $l_{1}$ is the first coordinate of $[l]$ and such that $(p)$ and $[l]$ are connected by an edge in $D(r, q)$. Analogously, if $[l] \in L$ and $p_{1} \in \mathbb{F}_{q}$ are fixed, the neighbor $(p)$ of $[l]$ is uniquely determined.

Proof. The first $r-1$ equations of (3) define a linear system for the unknown coordinates of $[l]$. If the equations and variables are written in the given order, the matrix corresponding to the resulting linear system of equations is a lower triangular matrix with non-zero elements in the diagonal. Hence, the matrix has full rank and by the basic laws of (finite) fields, the solution is unique. Exactly the same argumentation holds for the second claim of the lemma.

We are now ready to construct $G_{k}$ with large girth. We start with an arbitrary instance $G_{k}^{\prime}$ of the cluster tree which may have the minimum possible girth 4 . 
An elaboration of the construction of $G_{k}^{\prime}$ is deferred to Subsection 3.4. For now, we simply assume that $G_{k}^{\prime}$ exists. Both $G_{k}$ and $G_{k}^{\prime}$ are bipartite graphs with odd-level clusters in one set and even-level clusters in the other. Let $m$ be the number of nodes in the larger of the two partitions of $G_{k}^{\prime}$. We choose $q$ to be the smallest prime power greater than or equal to $m$. In both partitions $V_{1}\left(G_{k}^{\prime}\right)$ and $V_{2}\left(G_{k}^{\prime}\right)$ of $G_{k}^{\prime}$, we uniquely label all nodes $v$ with elements $c(v) \in \mathbb{F}_{q}$.

As already mentioned, $G_{k}$ is constructed as a subgraph of $D(r, q)$ for appropriate $r$ and $q$. We choose $q$ as described above and we set $r=2 k-4$ such that $g(D(r, q)) \geq 2 k+1$. Let $(p)=\left(p_{1}, \ldots\right)$ and $[l]=\left[l_{1}, \ldots\right]$ be two nodes of $D(r, q) .(p)$ and $[l]$ are connected by an edge in $G_{k}$ if and only if they are connected in $D(r, q)$ and there is an edge between nodes $u \in V_{1}\left(G_{k}^{\prime}\right)$ and $v \in V_{2}\left(G_{k}^{\prime}\right)$ for which $c(u)=p_{1}$ and $c(v)=l_{1}$. Finally, nodes without incident edges are removed from $G_{k}$.

Lemma 3.3. The graph $G_{k}$ constructed as described above is a cluster tree with the same degrees $\delta_{i}$ as in $G_{k}^{\prime}$. $G_{k}$ has at most $2 m q^{2 k-5}$ nodes and girth at least $2 k+1$.

Proof. The girth directly follows from the construction; removing edges cannot create cycles.

For the degrees between clusters, consider two neighboring clusters $C_{i}^{\prime} \subset V_{1}\left(G_{k}^{\prime}\right)$ and $C_{j}^{\prime} \subset$ $V_{2}\left(G_{k}^{\prime}\right)$ in $G_{k}^{\prime}$. In $G_{k}$, each node is replaced by $q^{2 k-5}$ new nodes. The clusters $C_{i}$ and $C_{j}$ consist of all nodes $(p)$ and $[l]$ which have their first coordinates equal to the labels of the nodes in $C_{i}^{\prime}$ and $C_{j}^{\prime}$, respectively. Let each node in $C_{i}^{\prime}$ have $\delta_{\alpha}$ neighbors in $C_{j}^{\prime}$, and let each node in $C_{j}^{\prime}$ have $\delta_{\beta}$ neighbors in $C_{i}^{\prime}$. By Lemma 3.2, nodes in $C_{i}$ have $\delta_{\alpha}$ neighbors in $C_{j}$ and nodes in $C_{j}$ have $\delta_{\beta}$ neighbors in $C_{i}$, too.

Remark: In [11], it has been shown that $D(r, q)$ is disconnected and consists of at least $q^{\left\lfloor\frac{r+2}{4}\right\rfloor}$ isomorphic components which the authors call $C D(r, q)$. Clearly, those components are valid cluster trees as well and we could use one of them for the analysis. As our asymptotic results remain unaffected by this observation, we continue to use $G_{k}$ as constructed above.

\subsection{Equality of Views}

In this subsection, we prove that two adjacent nodes in clusters $C_{0}$ and $C_{1}$ have the same view, i.e. within distance $k$, they see exactly the same topology $\mathcal{T}_{v, k}$. Consider a node $v \in G_{k}$. Given that $v$ 's view is a tree, we can derive its view-tree by recursively following all neighbors of $v$. The proof is largely based on the observation that corresponding subtrees occur in both node's view-tree.

Let $C_{i}$ and $C_{j}$ be adjacent clusters in $C T_{k}$ connected by $\ell\left(C_{i}, C_{j}\right)=\left(\delta_{l}, \delta_{l+1}\right)$, i.e. each node in $C_{i}$ has $\delta_{l}$ neighbors in $C_{j}$, and each node in $C_{j}$ has $\delta_{l+1}$ neighbors in $C_{i}$. When traversing a node's view-tree, we say that we enter cluster $C_{j}$ (resp., $C_{i}$ ) over link $\delta_{l}$ (resp., $\delta_{l+1}$ ) from cluster $C_{i}$ (resp., $C_{j}$ ). Furthermore, we make the following definitions:

Definition 3.4. The following nomenclature refers to subtrees in the view-tree of a node in $G_{k}$.

- $M_{i}$ is the subtree seen upon entering cluster $C_{0}$ over a link $\delta_{i}$.

- $B_{i, d, \lambda}$ is a subtree seen upon entering a cluster $C \in \mathcal{C} \backslash\left\{C_{0}\right\}$ over a link $\delta_{i}$, where $C$ is on level $\lambda$ and has depth $d$.

Definition 3.5. When entering subtree $B_{i, d, \lambda}$ from a cluster on level $\lambda-1(\lambda+1)$, we write $B_{i, d, \lambda}^{\uparrow}\left(B_{i, d, \lambda}^{\downarrow}\right)$. The predicate $\neg$ in $B_{i, d, \lambda}^{\neg}$ denotes that instead of $\delta_{i}$, the label of the link into this subtree is $\delta_{i}-1$.

The predicate $\neg$ is necessary when, after entering $C_{j}$ from $C_{i}$, we immediately return to $C_{i}$ on link $\delta_{i}$. In the view-tree, the edge used to enter $C_{j}$ connects the current subtree to its parent. Thus, this edge is not available anymore and there are only $\delta_{i}-1$ edges remaining to return to $C_{i}$. The predicates $\uparrow$ and $\downarrow$ describe from which "direction" a cluster has been entered. As the view-trees of nodes in $C_{0}$ and $C_{1}$ have to be absolutely identical for our proof to work, we must not neglect these admittedly tiresome details.

The following example should clarify the various definitions. Additionally, you may refer to the example of $G_{3}$ in Figure 3 on page 14.

Example 3.6. Consider $G_{1}$. Let $V_{C_{0}}$ and $V_{C_{1}}$ denote the view-trees of nodes in $C_{0}$ and $C_{1}$, respectively: 


$$
\begin{array}{ll}
V_{C_{0}}=B_{0,1,1}^{\uparrow} \cup B_{1,0,1}^{\uparrow} & V_{C_{1}}=B_{0,0,2}^{\uparrow} \cup M_{1} \\
B_{0,1,1}^{\uparrow}=B_{0,0,2}^{\uparrow} \cup M_{1}^{\uparrow} & B_{0,0,2}^{\uparrow}=B_{1,1,1}^{\downarrow,} \\
B_{1,0,1}^{\uparrow}=M_{2}^{\neg} & M_{1}^{\uparrow}=B_{0,1,1}^{\uparrow,,} \cup B_{1,0,1}^{\uparrow} \\
\cdots & \cdots
\end{array}
$$

We start the proof by giving a set of rules which describe the subtrees seen at a given point in the view-tree. We call these rules derivation rules because they allow us to derive the view-tree of a node by mechanically applying the matching rule for a given subtree.

Lemma 3.7. The following derivation rules hold in $G_{k}$ :

$$
\begin{aligned}
M_{i} & =\bigcup_{\substack{j=0 \ldots k \\
j \neq i-1}} B_{j, k-j, 1}^{\uparrow} \cup B_{i-1, k-i+1,1}^{\uparrow,\urcorner} \\
B_{i, d, 1}^{\uparrow} & =F_{\{i+1\}} \cup D_{\{\}} \cup M_{i+1}^{\urcorner} \\
B_{i, d, 1}^{\downarrow} & =F_{\{i-1, k-d+1\}} \cup D_{\{\}} \cup M_{k-d+1} \cup B_{i-1, d-1,2}^{\uparrow,\urcorner} \\
B_{i, d, \lambda}^{\uparrow} & =F_{\{i+1\}} \cup D_{\{i+1\}} \cup B_{i+1, d+1, \lambda-1}^{\downarrow,\urcorner}
\end{aligned}
$$

where $F$ and $D$ are defined as

$$
\begin{aligned}
F_{W} & :=\bigcup_{\substack{j=0 \ldots k-d+1 \\
j \notin W}} B_{j, d-1, \lambda+1}^{\uparrow} \\
D_{W} & :=\bigcup_{\substack{j=k-d+2 \ldots k \\
j \notin W}} B_{j, k-j, \lambda+1}^{\uparrow} .
\end{aligned}
$$

Proof. We first show the derivation rule for $M_{i}$. It can be seen in Example 3.6 that the rule holds for $k=1$. For the induction step, we build $C T_{k+1}$ from $C T_{k}$ as defined in Definition 3.1. $M^{(k)}$ is an inner cluster and therefore, one new cluster $B_{k+1,0,1}$ is added. The depth of all other subtrees increases by 1 and $M^{(k+1)}:=\bigcup_{j=0 \ldots k+1} B_{j, k-j, 1}^{\uparrow}$ follows. If we enter $M^{(k+1)}$ over link $\delta_{i}$, there will be only $\delta_{i-1}-1$ edges left to return to the cluster from which we had entered $C_{0}$. Consequently, the link $\delta_{i-1}$ features the $\neg$ predicate.

The remaining rules follow along the same lines. Let $C_{i}$ be a cluster with entry-link $\delta_{i}$ which was first created in $C T_{r}, r<k$ (Note that in $C T_{k}, r=k-d$ holds because each subtree increases its depth by one in each "round"). According to the second building rule of Definition 3.1, $r$ new neighboring clusters (subtrees) are created in $C T_{r+1}$. More precisely, a new cluster is created for all entry-links $\delta_{0} \ldots \delta_{r}$, except $\delta_{i}$. We call these subtrees fixed-depth subtrees $F$. If the subtree with root $C_{i}$ has depth $d$ in $C T_{k}$, the fixed-depth subtrees have depth $d-1$. In each $C T_{r^{\prime}}, r^{\prime} \in\{r+2, \ldots, k\}, C_{i}$ is an inner-cluster and hence, one new neighboring cluster with entry-link $\delta_{r^{\prime}}$ is created. We call these subtrees diminishingdepth subtrees $D$. In $C T_{k}$, each of these subtrees has grown to depth $k-r^{\prime}$.

We now turn our attention to the differences between the three rules. They stem from the exceptional treatment of level 1 , as well as the predicates $\uparrow$ and $\downarrow$. In $B_{i, d, 1}^{\uparrow}$, the link $\delta_{i+1}$ returns to $C_{0}$, but contains only $\delta_{i+1}-1$ edges in the view-tree. In $B_{i, d, 1}^{\downarrow}$, we have to consider two special cases. The first one is the link to $C_{0}$. For a cluster on level 1 with entry-link (from $C_{0}$ ) $i$, the equality $k=d+i$ holds and therefore, the link to $C_{0}$ is $\delta_{k-d+1}$ and thus, $M_{k-d+1}$ follows. Secondly, we write $\left.B_{i-1, d-1,2}^{\uparrow},\right\urcorner$ because there is one edge less leading back to the cluster where we had come from. (Note that since we entered the current cluster from a higher level, the link leading back to where we came from is $\delta_{i-1}$, instead of $\left.\delta_{i+1}\right)$. Finally in $B_{i, d, \lambda}^{\uparrow}$, we again have to treat the returning link $\delta_{i+1}$ specially.

Note that the general derivation rule for $B_{i, d, \lambda}^{\downarrow}$ is missing as we will not need it for the proof.

Next, we define the notion of r-equality. Intuitively, if two view-trees are $r$-equal, they have the same topology within distance $r$.

Definition 3.8. Let $V_{1}=\bigcup_{i=0 \ldots k} b_{i}$ and $V_{2}=$ $\bigcup_{i=0 \ldots k} b_{i}^{\prime}$ be view-trees; $b_{i}$ and $b_{i}^{\prime}$ are subtrees entered on link $\delta_{i}$. Then, $V_{1}$ and $V_{2}$ are r-equal if all corresponding subtrees are $(r-1)$-equal,

$$
V_{1} \stackrel{r}{=} V_{2} \Longleftarrow b_{i} \stackrel{r-1}{=} b_{i}^{\prime}, \forall i \in\{0, \ldots, k\} .
$$

Further, all subtrees are 0-equal: $B_{i, d, \lambda} \stackrel{0}{=} B_{i^{\prime}, d^{\prime}, \lambda^{\prime}}$ and $B_{i, d, \lambda} \stackrel{0}{=} M_{i^{\prime}}$ for all $i, i^{\prime}, d, d^{\prime}, \lambda$, and $\lambda^{\prime}$.

Using the notion of $r$-equality, it is now easy to define what we actually have to prove. We will show that in $G_{k}, V_{C_{0}} \stackrel{k}{=} V_{C_{1}}$ holds. This is equivalent to showing that each node in $C_{0}$ sees exactly the same topology within distance $k$ as its neighbor in $C_{1}$. We will now establish several helper lemmas. 
Lemma 3.9. Let $\beta$ and $\beta^{\prime}$ be sets of subtrees, and let $V_{v_{1}}=B_{i, d, x}^{\uparrow} \cup \beta$ and $V_{v_{2}}=B_{i, d, y}^{\uparrow} \cup \beta^{\prime}$ be two view-trees. Then, for all $x$ and $y$

$$
V_{v_{1}} \stackrel{r}{=} V_{v_{2}} \Longleftarrow \beta^{r-1}=\beta^{\prime} .
$$

Proof. Assume that the roots of the subtree of $V_{v_{1}}$ and $V_{v_{2}}$ are $C_{i}$ and $C_{j}$. The subtrees have equal depth and entry-link and they have thus grown identically. Hence, all paths which do not return to clusters $C_{i}$ and $C_{j}$ must be identical. Further, consider all paths which, after $s$ hops, return to $C_{i}$ and $C_{j}$ over link $\delta_{i+1}$. After these $s$ hops, they return to the original cluster and see views $V_{v_{1}}^{\prime}$ and $V_{v_{2}}^{\prime}$, differing from $V_{v_{1}}$ and $V_{v_{2}}$ only in the placement of the $\neg$ predicate. This does not affect $\beta$ and $\beta^{\prime}$ and therefore,

$$
V_{v_{1}} \stackrel{r}{=} V_{v_{2}} \Longleftarrow V_{v_{1}}^{\prime} \stackrel{r-s}{=} V_{v_{2}}^{\prime} \wedge \beta \stackrel{r-1}{=} \beta^{\prime}, s>1 .
$$

The same argument can be repeated until $r-s=0$ and because $V_{v_{1}}^{\prime} \stackrel{0}{=} V_{v_{2}}^{\prime}$, the lemma follows.

Lemma 3.10. Let $\beta$ and $\beta^{\prime}$ be sets of subtrees, and let $V_{v_{1}}=B_{i, d, x}^{\uparrow} \cup \beta$ and $V_{v_{2}}=B_{i, d^{\prime}, y}^{\uparrow} \cup \beta^{\prime}$ be two view-trees. Then, for all $x$ and $y$, and for all $r \leq$ $\min \left(d, d^{\prime}\right)$,

$$
V_{v_{1}} \stackrel{r}{=} V_{v_{2}} \Longleftarrow \beta^{r-1}=\beta^{\prime} .
$$

Proof. W.1.o.g, we assume $d^{\prime} \leq d$. In the construction process of $G_{k}$, the root clusters of $V_{v_{1}}$ and $V_{v_{2}}$ have been created in steps $k-d$ and $k-d^{\prime}$, respectively. By Definition 3.1, all subtrees with depth $d^{*}<d^{\prime}$ have grown identically in both views. The remaining subtrees of $V_{v_{2}}$ were all created in step $k-d^{\prime}+1$ and have depth $d^{\prime}-1$. The corresponding subtrees in $V_{v_{1}}$ have at least the same depth and hence, each pair of corresponding subtrees are $\left(d^{\prime}-1\right)$-equal. It follows that for $r \leq \min \left(d, d^{\prime}\right)$, the subtrees $B_{i, d, x}^{\uparrow}$ and $B_{i, d^{\prime}, y}^{\uparrow}$ are identical within distance $r$. Using the same argument as in Lemma 3.9 concludes the proof.

Figure 2 shows a part of the view-trees of nodes in $C_{0}$ and $C_{1}$ in $G_{3}$. The figure shows that the subtrees with links $\delta_{0}$ and $\delta_{2}$ cannot be matched directly to one another because of the different placement of the -1 . It turns out that this inherent difference appears in every step of our theorem. However, the
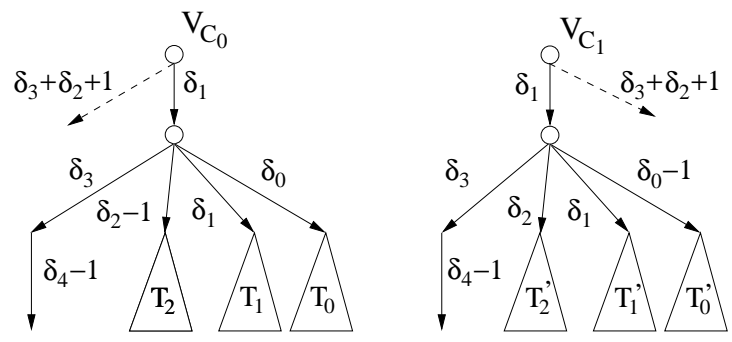

Figure 2: The view-trees $V_{C_{0}}$ and $V_{C_{1}}$ in $G_{3}$ seen upon using link $\delta_{1}$.

following lemma shows that the subtrees $T_{0}$ and $T_{2}$ ( $T_{0}^{\prime}$ and $T_{2}^{\prime}$ ) are equal up to the required distance and hence, nodes are unable to distinguish them. It is this crucial property of our cluster tree, which allows us to "move" the $\neg$ predicate between links $\delta_{i}$ and $\delta_{i+2}$ and enables us to derive the main theorem.

Lemma 3.11. Let $\beta$ and $\beta^{\prime}$ be sets of subtrees and let $V_{v_{1}}$ and $V_{v_{2}}$ be defined as

$$
\begin{aligned}
& V_{v_{1}}=M_{i}^{\urcorner} \cup B_{i-2, k-i, 2}^{\uparrow} \cup \beta \\
& V_{v_{2}}=M_{i} \cup B_{i-2, k-i, 2}^{\uparrow, \neg} \cup \beta^{\prime} \text {. }
\end{aligned}
$$

Then, for all $i \in\{2, \ldots, k\}$,

$$
V_{v_{1}} \stackrel{k-i}{=} V_{v_{2}} \Longleftarrow \beta^{\stackrel{k-i-1}{=}} \beta^{\prime} .
$$

Proof. Again, we make use of Lemma 3.7 to show that $M_{i}$ and $B_{i-2, k-i, 2}^{\uparrow}$ are $(k-i-1)$-equal. The claim then follows from the fact that the two subtrees are not distinguishable and the placement of the $\neg$ predicate is irrelevant.

$$
\begin{aligned}
M_{i}= & \bigcup_{\substack{j=0 \ldots k \\
j \neq i-1}} B_{j, k-j, 1}^{\uparrow} \cup B_{i-1, k-i+1,1}^{\uparrow, \neg} \\
B_{i-2, k-i, 2}^{\uparrow}= & \bigcup_{\substack{j=0 \ldots . .+1 \\
j \neq i-1}} B_{j, k-i-1,3}^{\uparrow} \cup \bigcup_{j=i+2 \ldots k} B_{j, k-j, 3}^{\uparrow} \\
& \cup \quad B_{i-1, k-i+1,1}^{\downarrow, \downarrow}
\end{aligned}
$$

For $j=\{0, \ldots, i-2, i, \ldots, k\}$, all subtrees are equal according to Lemmas 3.9 and 3.10. It remains to be shown that $B_{i-1, k-i+1,1}^{\uparrow} \stackrel{k-i-2}{=} B_{i-1, k-i+1,1}^{\downarrow}$. For that purpose, we plug $B_{i-1, k-i+1,1}^{\uparrow}$ and $B_{i-1, k-i+1,1}^{\downarrow}$ into 
Lemma 3.7 and show their equality using the derivation rules. Let $\beta$ be defined as $\beta:=F_{\{i-2, i\}} \cup D_{\{\}}$.

$$
\begin{aligned}
B_{i-1, k-i+1,1}^{\uparrow} & =F_{\{i\}} \cup D_{\{\}} \cup M_{i}^{\urcorner} \\
& =B_{i-2, k-i, 2}^{\uparrow} \cup M_{i}^{\urcorner} \cup \beta \\
B_{i-1, k-i+1,1}^{\downarrow} & =F_{\{i-2, i\}} \cup D_{\{\}} \cup M_{i} \cup B_{i-2, k-i, 2}^{\uparrow, \neg} \\
& =B_{i-2, k-i, 2}^{\uparrow,\urcorner} \cup M_{i} \cup \beta
\end{aligned}
$$

Again, if $M_{i}$ and $B_{i-2, k-i, 2}^{\uparrow}$ are $(k-i-3)$-equal, we can move the $\neg$ predicate because the subtrees are indistinguishable. Hence, we have to show $M_{i} \stackrel{k-i-3}{=} B_{i-2, k-i, 2}^{\uparrow}$. In the proof, we have reduced $V_{v_{1}} \stackrel{k-i}{=} V_{v_{2}}$ stepwise to an expression of diminishing equality conditions, i.e.

$$
\begin{aligned}
V_{v_{1}} \stackrel{k-i}{=} V_{v_{2}} & \Longleftarrow M_{i} \stackrel{k-i-1}{=} B_{i-2, k-i, 2}^{\uparrow} \\
& \Longleftarrow B_{i-1, k-i+1,1}^{\uparrow} \stackrel{k-i-2}{=} B_{i-1, k-i+1,1}^{\downarrow} \\
& \Longleftarrow M_{i} \stackrel{k-i-3}{=} B_{i-2, k-i, 2}^{\uparrow} .
\end{aligned}
$$

This process can be continued until either $B_{i-1, k-i+1,1}^{\uparrow} \stackrel{0}{=} B_{i-1, k-i+1,1}^{\downarrow}$ or $M_{i} \stackrel{0}{=} B_{i-2, k-i, 2}^{\uparrow}$ which is always true.

Finally, we are ready to prove the main theorem.

Theorem 3.12. Consider graph $G_{k}$. Let $V_{C_{0}}$ and $V_{C_{1}}$ be the view-trees of two adjacent nodes in clusters $C_{0}$ and $C_{1}$, respectively. Then, $V_{C_{0}} \stackrel{k}{=} V_{C_{1}}$.

Proof. Initially, each node in $C_{0}$ sees subtree $M_{*}$ and each node in $C_{1}$ sees $B_{*, k, 1}$ ( $*$ denotes that the subtree has not been entered on any link):

$$
\begin{array}{ll}
V_{C_{0}}: & M_{*}=\bigcup_{j=0 \ldots k} B_{j, k-j, 1}^{\uparrow} \\
V_{C_{1}}: & B_{*, k, 1}=\bigcup_{\substack{j=0 \ldots k \\
j \neq 1}} B_{j, k-j, 2}^{\uparrow} \cup M_{1} .
\end{array}
$$

It follows $V_{C_{0}} \stackrel{k}{=} V_{C_{1}} \Longleftarrow B_{1, k-1,1}^{\uparrow} \stackrel{k-1}{=} M_{1}$ because all other subtrees are $(k-1)$-equal by Lemma 3.9. Having reduced $V_{C_{0}} \stackrel{k}{=} V_{C_{1}}$ to $B_{1, k-1,1}^{\uparrow} \stackrel{k-1}{=} M_{1}$, we can further reduce it to $M_{2} \stackrel{k-2}{=} B_{2, k-2,1}^{\uparrow}$ :

$$
\begin{aligned}
M_{1} & =\bigcup_{j=1 \ldots k} B_{j, k-j, 1}^{\uparrow} \cup B_{0, k, 1}^{\uparrow,,} \\
B_{1, k-1,1}^{\uparrow} & =\quad B_{0, k-2,2}^{\uparrow} \cup B_{1, k-2,2}^{\uparrow} \cup D_{\{\}} \cup M_{2}^{\urcorner} \\
\begin{array}{c}
k=2 \\
\text { Lem. 3.11 }
\end{array} & B_{0, k-2,2}^{\uparrow, \uparrow} \cup B_{1, k-2,2}^{\uparrow} \cup D_{\{\}} \cup M_{2} .
\end{aligned}
$$

By Lemmas 3.9 and 3.10, all subtree are $(k-2)$ equal, except $B_{2, k-2,1}^{\uparrow}$ and $M_{2}$.

It is becoming clear that we can continue to reduce $V_{C_{0}} \stackrel{k}{=} V_{C_{1}}$ step by step in the same fashion until we reach 0 . For the induction step, we assume $V_{C_{0}} \stackrel{k}{=} V_{C_{1}} \Longleftarrow B_{r, k-r, 1}^{\uparrow} \stackrel{k-r}{=} M_{r}$ for $r<k$ and prove $V_{C_{0}} \stackrel{k}{=} V_{C_{1}} \Longleftarrow B_{r+1, k-r-1,1}^{\uparrow} \stackrel{k-r-1}{=} M_{r+1}$.

$$
\begin{aligned}
& M_{r}=\bigcup_{\substack{j=0 \ldots k \\
j \neq r-1}} B_{j, k-j, 1}^{\uparrow} \cup B_{r-1, k-r+1,1}^{\uparrow,\urcorner} \\
& B_{r, k-r, 1}^{\uparrow}=\bigcup_{j=0 \ldots r} B_{j, k-r-1,2}^{\uparrow} \cup D_{\{\}} \cup M_{r+1}^{\urcorner} \\
& \stackrel{k-r-1}{=} \operatorname{Lem.~3.11~}_{\substack{j=0 . . . r \\
j \neq r-1}} B_{j, k-r-1,2}^{\uparrow} \cup B_{r-1, k-r-1,2}^{\uparrow, \neg} \\
& \cup \bigcup_{j=r+2 \ldots k} B_{j, k-j, 2}^{\uparrow} \cup M_{r+1} .
\end{aligned}
$$

Apart from $M_{r+1}$ (resp,. $B_{r+1, k-r-1,1}^{\uparrow}$ ), all subtrees are $(k-r-1)$-equal by Lemmas 3.9 and 3.10. Since $M_{r+1}$ and $B_{r+1, k-r-1,1}^{\uparrow}$ are the only subtrees not being immediately matched, the induction step follows. For $r=k-1$, we get $V_{C_{0}} \stackrel{k}{=} V_{C_{1}} \Longleftarrow B_{k, 0,1}^{\uparrow} \stackrel{0}{=} M_{k}$, which concludes the proof because $B_{k, 0,1}^{\uparrow} \stackrel{0}{=} M_{k}$ is true.

Remark: As a side-effect, the proof of Theorem 3.12 has highlighted the fundamental significance of the critical path $P=\left(\delta_{1}, \delta_{2}, \ldots, \delta_{k}\right)$ in $C T_{k}$. After following path $P$, the view of a node $v \in C_{0}$ ends up in the leaf-cluster neighboring $C_{0}$ and sees $\delta_{i+1}$ neighbors. Following the same path, a node $v^{\prime} \in$ $C_{1}$ ends up in $C_{0}$ and sees $\sum_{j=0}^{i} \delta_{j}-1$ neighbors. There is no way to match these views. This inherent inequality is the underlying reason for the way $G_{k}$ is defined: It must be ensured that the critical path is at least $k$ hops long.

\subsection{Analysis}

In this subsection, we derive the lower bounds on the approximation ratio of $k$-local MVC algorithms. Let $O P T$ be an optimal solution for MVC and let $A L G$ be the solution computed by any algorithm. The main observation is that adjacent nodes in the clusters $C_{0}$ and $C_{1}$ have the same view and therefore, every algorithm treats nodes in both of the two 
clusters the same way. Consequently, $A L G$ contains a significant portion of the nodes of $C_{0}$, whereas the optimal solution covers the edges between $C_{0}$ and $C_{1}$ entirely by nodes in $C_{1}$.

Lemma 3.13. Let $A L G$ be the solution of any distributed, possibly randomized vertex cover algorithm which runs for at most $k$ rounds. When applied to $G_{k}$ as constructed in Subsection 3.2, in expectation, $A L G$ contains at least half of the nodes of $C_{0}$.

Proof. Let $v_{0} \in C_{0}$ and $v_{1} \in C_{1}$ be two arbitrary, adjacent nodes from $C_{0}$ and $C_{1}$. Further, let $p_{0}$ and $p_{1}$ denote the probabilities (as computed by the algorithm) that $v_{0}$ and $v_{1}$, respectively, end up in the vertex cover. The decision whether a given node $v$ enters the vertex cover depends solely on the topology $\mathcal{T}_{v, k}$, the labelling $\mathcal{L}\left(\mathcal{T}_{v, k}\right)$ and the randomness $\mathcal{R}\left(\mathcal{T}_{v, k}\right)$, which $v$ can collect during $k$ communication rounds. By Theorem 3.12, $v_{0}$ and $v_{1}$ see the same topologies, i.e. $\mathcal{T}_{v_{0}, k}=\mathcal{T}_{v_{1}, k}$. Imagine an adversary choosing the labelling of $G_{k}$; one possible strategy is to choose the labelling uniformly at random. In this case, the labelling $\mathcal{L}\left(\mathcal{T}_{v, k}\right)$ of $\mathcal{T}_{v, k}$ is chosen uniformly at random, as well. Because, $\mathcal{T}_{v_{0}, k}=\mathcal{T}_{v_{1}, k}$, the distributions of the random variables $\mathcal{L}\left(\mathcal{T}_{v_{0}, k}\right)$ and $\mathcal{L}\left(\mathcal{T}_{v_{1}, k}\right)$ are equal. Clearly, $\mathcal{R}\left(\mathcal{T}_{v_{0}, k}\right)$ and $\mathcal{R}\left(\mathcal{T}_{v_{1}, k}\right)$ have the same distribution, too. Hence, the views of $v_{0}$ and $v_{1}$ are equally distributed and thus, $p_{0}=p_{1}$.

We have chosen $v_{0}$ and $v_{1}$ such that they are neighbors in $G_{k}$. In order to obtain a feasible vertex cover, at least one of the two nodes has to be in it. This implies $p_{0}+p_{1} \geq 1$ and therefore $p_{0}=p_{1} \geq 1 / 2$. In other words, for all nodes in $C_{0}$, the probability to end up in the vertex cover is at least $1 / 2$. By the linearity of expectation, at least half of the nodes of $C_{0}$ are in the final vertex cover $A L G$ on average.

Lemma 3.13 gives a lower bound on the number of nodes chosen by any $k$-local MVC algorithm. In particular, we have that $\mathrm{E}[|A L G|] \geq\left|C_{0}\right| / 2=n_{0} / 2$. We do not know $O P T$, but since the nodes of cluster $C_{0}$ are not necessary to obtain a feasible vertex cover, the optimal solution is bounded by $|O P T| \leq n-n_{0}$. In the following, we define

$$
\delta_{i}:=\delta^{i}, \forall i \in\{0, \ldots, k+1\}
$$

for some value $\delta$.
Lemma 3.14. If $k+1<\delta$, the number of nodes $n$ of $G_{k}$ is

$$
n \leq n_{0}\left(1+\frac{k+1}{\delta-(k+1)}\right) .
$$

Proof. There are $n_{0}$ nodes in $C_{0}$. By (4), the number of nodes per cluster decreases for each additional level by a factor $\delta$. Hence, a cluster on level $l$ contains $n_{0} / \delta^{l}$ nodes. By the definition of $C T_{k}$, each cluster has at most $k+1$ neighboring clusters on a higher level. Thus, the number of nodes $n_{l}$ on level $l$ is upper bounded by

$$
n_{l} \leq(k+1)^{l} \cdot \frac{n_{0}}{\delta^{l}} .
$$

Summing up over all levels $l$ and interpreting the sum as a geometric series, we obtain

$$
\begin{aligned}
n & \leq n_{0} \cdot \sum_{i=0}^{k+1}\left(\frac{k+1}{\delta}\right)^{l} \leq n_{0} \cdot \sum_{i=0}^{\infty}\left(\frac{k+1}{\delta}\right)^{l} \\
& =n_{0}+n_{0}\left(\frac{k+1}{\delta}\right)\left(\frac{1}{1-\frac{k+1}{\delta}}\right) \\
& =n_{0}\left(1+\frac{k+1}{\delta-(k+1)}\right) .
\end{aligned}
$$

It remains to determine the relationship between $\delta$ and $n_{0}$ such that $G_{k}$ can be realized as described in Subsection 3.2. There, the construction of $G_{k}$ with large girth is based on a smaller instance $G_{k}^{\prime}$ where girth does not matter. Using (4) (i.e. $\delta_{i}:=\delta^{i}$ ), we can now tie up this loose end and describe how to obtain $G_{k}^{\prime}$. The number of nodes per cluster decreases by a factor $\delta$ on each level of $C T_{k}$. Including $C_{0}, C T_{k}$ consists of $k+2$ levels. The maximum number of neighbors inside a leaf-cluster is $\delta^{k}$. Hence, we can set the sizes of the clusters on the outermost level $k+1$ to be $\delta^{k}$. This implies that the size of a cluster on level $l$ is $\delta^{2 k+1-l}$. Particularly, the size of $C_{0}^{\prime}$ at level 0 in $G_{k}^{\prime}$ is $n_{0}^{\prime}=\delta^{2 k+1}$. Let $C_{i}$ and $C_{j}$ be two adjacent clusters with $\ell\left(C_{i}, C_{j}\right)=\left(\delta^{i}, \delta^{i+1}\right) . C_{i}$ and $C_{j}$ can simply be connected by as many complete bipartite graphs $K_{\delta^{i}, \delta^{i+1}}$ as necessary.

If we assume that $k+1 \leq \delta / 2$, we have $n \leq$ $2 n_{0}$ by Lemma 3.14. Applying the construction of Subsection 3.2, we get $n_{0} \leq n_{0}^{\prime} \cdot\left\langle n^{\prime}\right\rangle^{2 k-5}$, where 
$\left\langle n^{\prime}\right\rangle$ denotes the smallest prime power larger than or equal to $n^{\prime}$, i.e. $\left\langle n^{\prime}\right\rangle<4 n_{0}^{\prime}$. Putting all together, we get

$$
n_{0} \leq\left(4 n_{0}^{\prime}\right)^{2 k-4} \leq 4^{2 k-4} \delta^{4 k^{2}} .
$$

Theorem 3.15. There are graphs $G$, such that in $k$ communication rounds, every distributed algorithm for the minimum vertex cover problem on $G$ has approximation ratios at least

$$
\Omega\left(\frac{n^{c / k^{2}}}{k}\right) \text { and } \Omega\left(\frac{\Delta^{1 / k}}{k}\right)
$$

for some constant $c \geq 1 / 4$, where $n$ and $\Delta$ denote the number of nodes and the highest degree in $G$, respectively.

Proof. We can choose $\delta \geq 4^{-1 /(2 k)} n_{0}^{1 /\left(4 k^{2}\right)}$ due to Inequality (5). Finally, using Lemmas 3.13 and 3.14, the approximation ratio $\alpha$ is at least

$$
\begin{aligned}
\alpha & \geq \frac{n_{0} / 2}{n-n_{0}} \geq \frac{n_{0} / 2 \cdot \delta / 2}{n_{0} \cdot(k+1)}=\frac{\delta}{4(k+1)} \\
& \geq \frac{(n / 2)^{1 /\left(4 k^{2}\right)}}{4^{1+1 /(2 k)}(k+1)} \in \Omega\left(\frac{n^{1 /\left(4 k^{2}\right)}}{k}\right) .
\end{aligned}
$$

The second lower bound follows from $\Delta=\delta^{k+1}$.

Theorem 3.16. In order to obtain a polylogarithmic or even constant approximation ratio, every distributed algorithm for the MVC problem requires at least $\Omega\left(\sqrt{\frac{\log n}{\log \log n}}\right)$ and $\Omega\left(\frac{\log \Delta}{\log \log \Delta}\right)$ communication rounds.

Proof. We set $k=\beta \sqrt{\log n / \log \log n}$ for an arbitrary constant $\beta>0$. When plugging this into the first lower bound of Theorem 3.15, we get the following approximation ratio $\alpha$ :

$$
\alpha \geq \gamma n^{\frac{c \log \log n}{\beta^{2} \log n}} \cdot \frac{1}{\beta} \sqrt{\frac{\log \log n}{\log n}}
$$

where $\gamma$ is the hidden constant in the $\Omega$-notation. For the logarithm of $\alpha$, we get

$$
\begin{aligned}
\log \alpha & \geq \frac{c \log \log n}{\beta^{2} \log n} \cdot \log n-\frac{1}{2} \cdot \log \log n-\log \beta \\
& =\left(\frac{c}{\beta^{2}}-\frac{1}{2}\right) \cdot \log \log n-\log \beta .
\end{aligned}
$$

and therefore

$$
\alpha \in \Omega\left(\log (n)^{\left(\frac{c}{\beta^{2}}-\frac{1}{2}\right)}\right) .
$$

By choosing an appropriate $\beta$, we can determine the exponent of the above expression. For every polylogarithmic term $\alpha(n)$, there is a constant $\beta$ such that the above expression is at least $\alpha(n)$ and hence, the first lower bound of the theorem follows.

The second lower bound follows from an analogous computation by setting $k=\beta \log \Delta / \log \log \Delta$.

Remark: By defining $\delta_{i}:=\delta^{i}, \quad i \in\{0, \ldots, k\}$ and $\delta_{k+1}:=\delta^{k+1 / 2}$ (instead of $\delta^{k+1}$ ), we obtain slightly stronger approximation lower bounds of

$$
\Omega\left(n^{c / k^{2}}-k\right) \text { and } \Omega\left(\Delta^{c^{\prime} / k}-k\right) .
$$

The bounds of (6) clearly do not suffice to improve the results of Theorem 3.16.

\section{Reductions}

Using the lower bound for vertex cover, we can obtain lower bounds for several other classical graph problems. In this section, we give time lower bounds for the construction of maximal matchings and maximal independent sets as well as for the approximation of minimum dominating set.

A maximal matching (MM) of a graph $G$ is a maximal set of edges which do not share common endpoints. Hence, a MM is a set of non-adjacent edges of $G$ such that all edges in $E(G) \backslash M M$ have a common end-point with an edge in MM. A maximal independent set (MIS) is a maximal set of non-adjacent nodes, i.e. all nodes not in the MIS are adjacent to some node of the MIS. The best known lower bound for the distributed computation of a MM or a MIS is $\Omega\left(\log ^{*} n\right)$ which holds for rings [12]. Based on Theorem 3.16, we get the following stronger lower bounds.

Theorem 4.1. There are graphs $G$ on which every distributed, possibly randomized algorithm requires time $\Omega\left(\sqrt{\frac{\log n}{\log \log n}}\right)$ and $\Omega\left(\frac{\log \Delta}{\log \log \Delta}\right)$ to compute a maximal matching. The same lower bounds hold for the construction of maximal independent sets. 
Proof. It is well known that the set of all endpoints of the edges of a MM form a 2-approximation for MVC. This simple 2-approximation algorithm is commonly attributed to Gavril and Yannakakis. The lower bound for the construction of a MM therefore directly follows from Theorem 3.16.

For the MIS problem, consider the line graph $L\left(G_{k}\right)$ of $G_{k}$. The nodes of a line graph $L(G)$ of $G$ are the edges of $G$. Two nodes in $L(G)$ are connected by an edge whenever the two corresponding edges in $G$ are incident to the same node. The MM problem on a graph $G$ is equivalent to the MIS problem on $L(G)$. Further, if the real network graph is $G$, $k$ communication rounds on $L(G)$ can be simulated in $k+\mathrm{O}(1)$ communication rounds on $G$. Therefore, the times $t$ to compute a MIS on $L\left(G_{k}\right)$ and $t^{\prime}$ to compute a MM on $G_{k}$ can only differ by a constant, $t \geq t^{\prime}-\mathrm{O}(1)$. Let $n^{\prime}$ and $\Delta^{\prime}$ denote the number of nodes and the maximum degree of $G_{k}$, respectively. The number of nodes $n$ of $L\left(G_{k}\right)$ is less than $n^{\prime 2} / 2$, the maximum degree $\Delta$ of $G_{k}$ is less than $2 \Delta^{\prime}$. Because $n^{\prime}$ only appears as $\log n^{\prime}$, the power of 2 does not hurt and the theorem holds $\left(\log n=\Theta\left(\log n^{\prime}\right)\right)$.

We conclude this section by considering the problem of approximating the minimum dominating set (MDS) problem. A dominating set $S$ is a subset of the nodes of a graph $G$ such that all nodes of $G$ are either in $S$ or they have a neighbor in $S$. In a nondistributed setting, MDS in equivalent to the general minimum set cover problem ${ }^{2}$ whereas MVC is a special case of set cover which can be approximated much better. It is therefore not surprising that in a distributed environment, MDS is strictly harder than MVC, too. In the following, we show that this intuitive fact can be formalized.

Theorem 4.2. There are graphs $G$, such that in $k$ communication rounds, every distributed algorithm for the minimum dominating set problem on $G$ has approximation ratios at least $\Omega\left(n^{c / k^{2}} / k\right)$ and $\Omega\left(\Delta^{1 / k} / k\right)$ for some constant $c$, where $n$ and $\Delta d e$ note the number of nodes and the highest degree in $G$, respectively.

\footnotetext{
${ }^{2}$ There exist approximation preserving reductions in both directions.
}

Proof. We show that every MVC instance can be seen as a MDS instance with the same locality. Let $G^{\prime}=\left(V^{\prime}, E^{\prime}\right)$ be a graph for which we want to solve MVC. We construct the corresponding dominating set graph $G=(V, E)$ as follows. For every node and for every edge in $G^{\prime}$, there is a node in $G$. We call nodes $v_{n} \in V$ corresponding to nodes $v^{\prime} \in V^{\prime}$ $n$-nodes, and nodes $v_{e} \in V$ corresponding to edges $e^{\prime} \in E^{\prime}$ e-nodes. Two $n$-nodes are connected by an edge if and only if they are adjacent in $G^{\prime}$. An $n$ node $v_{n}$ and an $e$-node $v_{e}$ are connected exactly if the corresponding node and edge are incident in $G^{\prime}$. There are no edges between two $e$-nodes. Clearly, the localities of $G^{\prime}$ and $G$ are the same, i.e. $k$ communication rounds on one of the two graphs can be simulated by $k+\mathrm{O}(1)$ rounds on the other graph. Let $C$ be a feasible vertex cover for $G^{\prime}$. We claim that all nodes of $G$ corresponding to nodes in $C$ form a valid dominating set on $G$. By definition, all $e$-nodes are covered. The remaining nodes of $G$ are covered because for a given graph, a valid vertex cover is a valid dominating set as well. Therefore, the optimal dominating set on $G$ is at most as big as the optimal vertex cover on $G^{\prime}$. There also exists a transformation in the other direction. Let $D$ be a valid dominating set on $G$. If $D$ contains an $e$-node $v_{e}$, we can replace $v_{e}$ by one of its two neighbors. The size of $D$ remains the same and all three nodes covered (dominated) by $v_{e}$ are still covered. By this, we get a dominating set $D^{\prime}$ which has the same size as $D$ and which consists only of $n$-nodes. Because $D^{\prime}$ dominates all $e$-nodes, the nodes of $G^{\prime}$ corresponding to $D^{\prime}$ form a valid vertex cover. Thus, MDS on $G$ is exactly as hard as MVC on $G^{\prime}$ and the theorem follows from Theorem 3.15 .

Corollary 4.3. To obtain a polylogarithmic (or constant) approximation ratio for minimum dominating set, there are graphs on which every distributed algorithm needs time $\Omega\left(\sqrt{\frac{\log n}{\log \log n}}\right)$ and $\Omega\left(\frac{\log \Delta}{\log \log \Delta}\right)$. Proof. The corollary is a direct consequence of Theorem 4.2 and the proof of Theorem 3.16.

Remark: Note that in the above corollary, we give a time lower bound for constant MDS approximation although it has been shown that MDS cannot be approximated better than $\ln \Delta$ unless $\mathrm{NP} \subseteq$ 
$\operatorname{DTIME}\left(n^{\mathrm{O}(\log \log n)}\right)$ [3]. Because local computation is for free in our model, however, it is theoretically possible to get a constant-factor approximation for MDS.

\section{Conclusions}

As distributed systems grow larger, it is becoming increasingly vital to design algorithms which do not need to maintain full information about the network. We have shown locality-imposed restrictions on the approximability and computability of a number of distributed problems. Comparing with the respective upper bounds, some of our lower bounds are near tight. In $k$ rounds, MVC can be approximated by a factor $\mathrm{O}\left(\Delta^{1 / k}\right)$; MDS can be approximated by factors $\mathrm{O}\left(\Delta^{c / \sqrt{k}}\right)$ and $\mathrm{O}\left(n^{c / k}\right)$, respectively [8]. The best known algorithms for maximal matching and maximal independent set need time $\mathrm{O}(\log n)[6,13]$. 


\section{References}

[1] Y. Afek, S. Kutten, and M. Yung. The Local Detection Paradigm and its Applications to SelfStabilization. Theoretical Computer Science, 186(12):199-229, 1997.

[2] R. Cole and U. Vishkin. Deterministic Coin Tossing with Applications to Optimal Parallel List Ranking. Information and Control, 70(1):32-53, 1986.

[3] U. Feige. A Threshold of $\ln \mathrm{n}$ for Approximating Set Cover. Journal of the ACM (JACM), 45(4):634-652, 1998.

[4] F. Fich and E. Ruppert. Hundreds of impossibility results for distributed computing. Distrib. Comput., 16(2-3):121-163, 2003.

[5] M. J. Fischer, N. A. Lynch, and M. S. Paterson. Impossibility of Distributed Consensus With One Faulty Process. J. ACM, 32(2):374-382, 1985.

[6] A. Israeli and A. Itai. A Fast and Simple Randomized Parallel Algorithm for Maximal Matching. Information Processing Letters, 22:77-80, 1986.

[7] F. Kuhn and R. Wattenhofer. Constant-Time Distributed Dominating Set Approximation. In Proc. of the 22 ${ }^{\text {nd }}$ Annual ACM Symp. on Principles of Distributed Computing (PODC), pages 25-32, 2003.

[8] F. Kuhn and R. Wattenhofer. Distributed Combinatorial Optimization. Technical Report 426, ETH Zurich, Dept. of Computer Science, 2003.

[9] L. Lamport, R. Shostak, and M. Pease. The Byzantine Generals Problem. ACM Trans. Program. Lang. Syst., 4(3):382-401, 1982.

[10] F. Lazebnik and V. A. Ustimenko. Explicit Construction of Graphs with an Arbitrary Large Girth and of Large Size. Discrete Applied Mathematics, 60(1-3):275-284, 1995.

[11] F. Lazebnik, V. A. Ustimenko, and A. J. Woldar. A New Series of Dense Graphs of High Girth. Bulletin of the American Mathematical Society (N.S.), 32(1):73-79, 1995.

[12] N. Linial. Locality in Distributed Graph Algorithms. SIAM Journal on Computing, 21(1):193-201, 1992.

[13] M. Luby. A Simple Parallel Algorithm for the Maximal Independent Set Problem. SIAM Journal on Computing, 15:1036-1053, 1986.

[14] A. Mayer, M. Naor, and L. Stockmeyer. Local Computations on Static and Dynamic Graphs. In Proc. of IEEE $3^{\text {rd }}$ Israeli Symp. on Theory of Computing and Systems (ISTCS), pages 268-278, 1995.
[15] M. Naor and L. Stockmeyer. What Can Be Computed Locally? In Proc. of the $25^{\text {th }}$ Annual ACM Symp. on Theory of Computing (STOC), pages 184193, 1993.

[16] D. Peleg. Distributed Computing: A LocalitySensitive Approach. SIAM, 2000. 

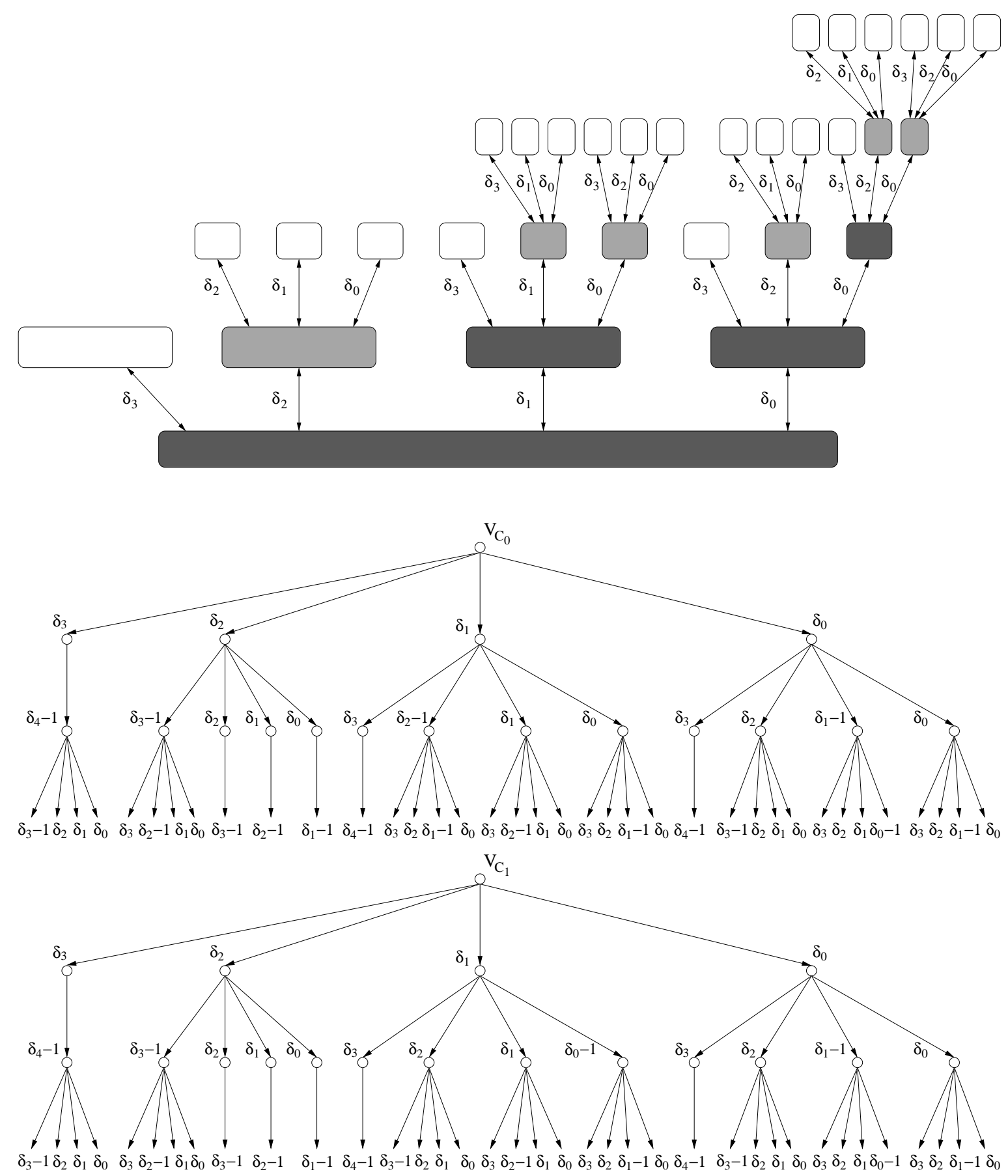

Figure 3: The Cluster Tree $C T_{3}$ and the corresponding view-trees of nodes in $C_{0}$ and $C_{1}$. The cluster trees $C T_{1}$ and $C T_{2}$ are shaded dark and light, respectively. The labels of the arcs of the cluster tree represent the number of neighbors of nodes of the lower-level cluster in the neighboring higher-level cluster. The labels of the reverse links are omitted. In the view-trees, an arc labelled with $\delta_{i}$ stands for $\delta_{i}$ edges, all connecting to identical subtrees. 